\title{
VIOLENCIA ESCOLAR, PATRONES DE GÉNERO Y DERECHOS FUNDAMENTALES. UNA REFLEXIÓN A PARTIR DEL «CASO CARLA»
}

\author{
MARÍA L. VALVIDARES SUÁREZ \\ Universidad de Oviedo
}

\begin{abstract}
A la memoria de Carla y su familia, y de quienes luchan contra el acoso escolar, con el único objetivo de reflexionar sobre esta «conspiración del silencio».
\end{abstract}

Recibido 26-03-2015

Aceptado 14-05-2015

\section{Resumen}

El objeto de este artículo es reflexionar, a partir de un reciente caso de acoso escolar, sobre los problemas que presenta la respuesta jurídica frente al acoso. El trabajo centra su atención en el derecho a la educación, como derecho que ha de vertebrar la reacción de la comunidad frente al acoso escolar, al ser la herramienta para formar personas que respeten los principios democráticos de convivencia frente a las situaciones de vulnerabilidad por razón de edad, género y orientación sexual, entre otros factores.

Palabras clave: acoso escolar, ciber-acoso, derechos fundamentales, género, igualdad, derecho a la educación

\begin{abstract}
Moving from a recent case of bullying, this article tries to reflect on the problems deriving from the legal reaction. It focuses on the right to education, as the right that has to guide community's reaction towards bullying, since it is the tool to make people respect democratic principles of cohabitation facing vulnerability situations due to minority, gender and sexual orientation, among others.
\end{abstract}

Key-words: bullying, cyber-bullying, fundamental rights, gender, equality, right to education 



\section{Introducción. Exposición del caso de Carla}

En 2013, Carla, una adolescente de 14 años, se suicidó. Carla estaba siendo víctima de acoso escolar, tanto dentro del Instituto como a través de diversas redes sociales. Se acusó a dos compañeras de Carla del delito de inducción al suicidio, pero la Fiscalía archivó el caso. Asistida por una nueva defensa letrada, la madre de Carla denunció a las dos alumnas por vulneración del derecho a la integridad moral, hecho por el que fueron condenadas a realizar cuatro meses de tareas socio-educativas ${ }^{2}$. Una ulterior cuestión pendiente de resolución es la relativa a la responsabilidad civil subsidiaria de la institución educativa. Así ha sucedido en otros casos de acoso escolar, en los que se ha puesto en cuestión la actitud indolente del centro frente a estos comportamientos, como se indicará en su momento. Un problema adicional, presente en el caso de Carla -y cada vez más común- lo plantea el hecho de que gran parte de los comportamientos de acoso se canalizaran a través de redes sociales. ¿Implica una exclusión de la responsabilidad del centro educativo? ¿Deja de ser una situación en la que debe intervenir?

El caso de Carla, como el de muchos otros jóvenes que sufren acoso escolar, nos obliga a reflexionar. El objeto de este artículo es profundizar en algunas cuestiones de carácter jurídico relacionadas con los comportamientos y reacciones frente al acoso escolar. En primer lugar se abordarán las cuestiones conceptuales, toda vez que no existe una definición legal del acoso escolar (como sí sucede, por ejemplo, con la violencia de género), realizando algunos matices relativos a la presencia de las nuevas tecnologías y de los factores vinculados al género, ambos presentes en el caso de Carla. Estas reflexiones permitirán entroncar con la cuestión de la regulación legal y abordar las dificultades que esta presenta para dar una respuesta adecuada a las particularidades del acoso escolar. A continuación, se pondrá el acento en los derechos

1. Este artículo es en gran parte deudor de las reflexiones de la abogada Leticia de La Hoz -abogada de la familia de Carla, que se ha convertido en un referente en la defensa jurídica del acoso escolar- sobre muchas de las lagunas y problemas de índole prácticojurídica que se presentan al abordar estos casos.

2. Sentencia núm.380/14, Juzgado de menores de Oviedo, de 30 de diciembre de 2014.

Feminismo/s 25, junio 2015, pp. 13-34 
fundamentales de los menores que se ven afectados, especialmente los de las víctimas, pero también de quienes realizan las agresiones, toda vez que el análisis de las situaciones de acoso y la reacción al mismo debería vertebrarse a partir del derecho constitucional a la educación.

\section{Delimitación conceptual: acoso, ciber-acoso y SRGBV}

La violencia en el ámbito escolar es un fenómeno antiguo, pero su tratamiento como un problema que debe ser erradicado es relativamente reciente. La doctrina considera que los primeros estudios sobre el tema se deben al psicólogo nórdico Dan Olweus, y datan de la década de los años setenta. El fenómeno fue cobrando cada vez mayor interés para varias disciplinas, por lo que tanto la psicología, la psiquiatría, la educación y la pedagogía e incluso la pediatría han reflexionado al respecto ${ }^{3}$. Sin embargo, la atención prestada desde el ámbito jurídico es menor. La propia Fiscalía General del Estado lo define como un fenómeno «oculto, que pese a haber estado presente desde siempre en las relaciones entre los menores...no ha generado estudios, reflexiones o reacciones ni desde el ámbito académico ni desde las instancias oficiales ${ }^{4}$, llegando a hablar la doctrina de una «conspiración del silencio» ${ }^{5}$.

Para abordar correctamente el análisis de los derechos en presencia, y dada la complejidad de una situación que normalmente incluye una multiplicidad de factores y conductas, es oportuno realizar una aproximación conceptual básica. En primer lugar, del propio concepto de acoso escolar difundido en los últimos años a través del término anglosajón de bullying ${ }^{6}$. A continuación,

3. Uno de los más recientes, que además resulta especialmente relevante al centrarse en el ámbito territorial del Principado de Asturias, es el de ÁlvarEZ-GARCía, David; DoBARRO, Alejandra; ÁlvareZ, Luis; NúÑEZ, José C.; RODRíGUEZ, Celestino. «La violencia escolar en los centros de educación secundaria de Asturias desde la perspectiva del alumnado». Educación XXI, 17.2 (2014), pp. 337-360.

4. Instrucción 10/2005 sobre el tratamiento del acoso escolar desde el sistema de justicia juvenil. Disponible en la web de la Fiscalía General del Estado: <www.fiscal.es> (consultado el 18-03-2015)

5. DíAZ-Aguado Jalón, María José. «La violencia entre iguales en la adolescencia y su prevención desde la escuela». Psicothema 4, 17 (2005), p. 550.

6. En términos generales se usará el término de acoso escolar, toda vez que la palabra anglosajona bullying, que cada vez goza de mayor aceptación, parece en principio referida al fenómeno del «matonismo», ligada, por tanto, sobre todo al uso de la fuerza y la violencia física. El concepto de acoso o de violencia escolar permite incluir una variedad de comportamientos relevantes para un fenómeno de perfiles complejos, como pueden ser no solo la violencia verbal, sino también las exclusiones, el hostigamiento, las «incivilidades y las microviolencias». Cfr. BLAYA, Catherine; DEBARBIEUX, Eric y LUCAS, Beatriz. "La violencia hacia las mujeres y hacia otras personas percibidas como distintas a la norma dominante: el caso de los centros educativos». Revista de Educación.

Feminismo/s 25, junio 2015, pp. 13-34 
de una modalidad relativamente novedosa, derivada de la integración de las redes sociales tanto en las relaciones personales cotidianas como en la propia dinámica escolar ${ }^{7}$, que se viene conociendo como «ciber-acoso», «ciber-maltrato» o cyberbullying. ${ }^{8}$. Y, por último, del concepto de «violencia de género relacionada con la escuela», denominación que traduce la categoría acuñada internacionalmente como School-related Gender-Based Violence, y que se conoce por su acrónimo SRGBV.

El concepto de acoso escolar se enmarca en el contexto de comportamientos de violencia en las relaciones entre iguales (peer), no en el sentido de ausencia de dominio sino de relaciones de horizontalidad (es decir, en el alumnado) para diferenciarla de las situaciones de violencia escolar verticales (entre profesorado y alumnado). En este sentido, la doctrina suele caracterizar el acoso o bullying por: (1) la heterogeneidad de conductas (burlas, amenazas, intimidaciones, agresiones físicas, aislamiento sistemático, insultos); (2) originar problemas que normalmente se repiten y prolongan en el tiempo; (3) implicar abuso de poder por parte de uno o varios alumnos frente a una víctima que no puede salir por si sola de su situación de indefensión; y (4) el mantenimiento de la situación debido a la ignorancia o la pasividad de quienes rodean a víctimas y agresores ${ }^{9}$.

342 (2007), p. 68. Respecto de las dificultades terminológicas, también se pronuncia el informe del Defensor del Pueblo: «Violencia escolar: el maltrato entre iguales en la Educación Secundaria Obligatoria 1999-2006», Madrid, 2007, pp. 18-19.

7. LI, Qing. «New bottle but old wine: A research of cyberbullying in schools». Computers in Human Behaviour 23 (2007), pp. 1778-1779. Véase el Informe Ejecutivo de 2012 del Instituto Vasco de Evaluación e Investigación Educativa sobre el Maltrato entre iguales en Euskadi, para una aproximación reciente a las cifras de impacto de cyberbulling.

8. A pesar de la novedad, el fenómeno del ciber-acoso ha recibido bastante atención. Véanse, a modo de ejemplo: Ortega, Rosario; CAlmaestra, Juan; Mora, Joaquin. «Cyberbullying». International Journal of Psychology and Psychological Therapy 2, 8 (2008), pp. 183-192; TOKUNAGA, Robert S. «Following you home from school: A critical review and synthesis of research on cyberbullying victimization», Computers in Human Behaviour 26 (2010), pp. 277-287; CALVETE, Esther; Orue, Izaskum; EstÉVEZ, Ana; Villardón, Lourdes; PADILla, Patricia. «Cyberbullying in adolescents: Modalities and aggressors' profile». Computers in Human Behaviour 26 (2010), pp. 1128-1135. Una investigación en el ámbito asturiano, relevante por el caso de estudio que ha dado origen a este artículo, se recoge en ÁlVAREZ-GARCÍA, David; NÚÑEZ-PÉREZ, José Carlos; Álvarez-PÉrez, Luis; Dobarro-GonZÁlez, Alejandra; RodrígueZ-PÉrez, Celestino; GONZÁleZ-CASTRO, Paloma. "Violencia a través de las tecnologías de la información y la comunicación en estudiantes de secundaria». Anales de psicología 27, 1 (2011), pp. 221-231.

9. DíAz-Aguado, María José. «La violencia entre iguales en la adolescencia...», op. cit., p. 549. Véase para ulteriores referencias bibliográficas el trabajo de CÁCERES, María Pilar; ALONSO, Santiago; GARROTE, Daniel. «Aportaciones para el estudio de 
El ciber-acoso puede definirse como el «daño repetido e intencionado ocasionado a través de medios electrónicos como teléfonos móviles o internet». Es fundamental resaltar que se diferencia del acoso tradicional por tres factores principales: (1) no cesa al salir del centro escolar; (2) puede implicar a grupos más numerosos de personas y (3) invisibiliza a los agresores, reforzando una falsa sensación de impunidad ${ }^{10}$. El tercer factor ha sido particularmente relevante en el caso de estudio, a través de la red ask.fm que permite una cierta participación anónima, y que está canalizando conductas de ciberacoso que ya han sido denunciadas ${ }^{11}$.

Por último, la violencia de género relacionada con la escuela se define como

los actos de violencia sexual, física o psicológica infligida sobre los menores dentro de la escuela y del entorno escolar, debido a estereotipos y roles o normas atribuidas a los mismos a causa de su identidad sexual o de género. Asimismo, también se refiere a las diferencias entre chicas y chicos en su experiencia relativa a la violencia y a su vulnerabilidad ante la misma ${ }^{12}$.

Estos conceptos deben ayudarnos a reflexionar sobre las razones por las cuales los centros de enseñanza no pueden permanecer pasivos, desentendiéndose de la situación de acoso pensando -en el mejor de los casos- que es mejor no intervenir al tratarse de un asunto "entre iguales», o -en el peor- que se trata de procesos normales en el tránsito a la madurez que no tienen trascendencia. Ambas posturas son inaceptables, tanto desde postulados pedagógicos como jurídicos. Es fundamental no perder de vista que los menores son titulares de derechos fundamentales protegidos a nivel constitucional e internacional. Esta perspectiva guía estas reflexiones y justifica la imperiosa necesidad de que el ámbito jurídico muestre mayor atención a lo que sucede en el educativo.

la violencia escolar desde una perspectiva interdisciplinar desde el ámbito universitario, escolar, familiar y social». Ensayos 16 (2008), pp. 221-236.

10. FÉlix-Mateo, Vicente; Soriano-Ferrer, Manuel; Godoy-Mesas, Carmen; SanchoVICENTE, Sonia. «El ciberacoso en la enseñanza obligatoria». Aula Abierta 38, 1 (2010), pp. $47-48$.

11. De hecho, esta plataforma anunció un cambio en sus protocolos de funcionamiento con el objeto de incorporar la posibilidad de denunciar por acoso los comentarios de los usuarios, después de que en el mismo año 2013 se suicidara una adolescente británica que había sufrido cyberbullying.

12. Extraído de Greene, M.; Robles, O.; Stout, K. y SuvilaAKSO, T. «A girls right to learn without fear: working to end gender-based violence at school». Woking: Plan international, 2013, disponible en: http://plan-international.org/about-plan/resources/publications/campaigns/summary-a-girls-right-to-learn-without-fear/working-together-endviolence, consultado el 11-03-2015.

Feminismo/s 25, junio 2015, pp. 13-34 


\section{El acoso escolar y su problemática regulación legal}

El punto de partida al reflexionar sobre la respuesta legal frente al acoso escolar es la inexistencia de un tipo penal específico, por lo que debe indagarse una a una sobre las conductas realizadas y buscar su encaje en los tipos penales generales ${ }^{13}$. Con ello se pone de relieve la dificultad -por no decir la incapacidad- de que el sistema penal ofrezca una respuesta adecuada, tanto por la inexistencia de una regulación específica como por la particularidad del fenómeno del acoso escolar ${ }^{14}$.

Como la propia Fiscalía pone de relieve en la Instrucción 10/2005 citada, la Recomendación núm. 702 del Comité de Derechos del Niño de la ONU señala que el castigo que pueda proceder de la justicia (penal) juvenil debe ser subsidiario y no el método principal de reacción frente al acoso. No debe sorprender que esta reflexión se enmarque en los derechos del niño, toda vez que en los casos de acoso escolar hablamos bien de menores de entre 14 y 18 años, por tanto sometidos en principio a un sistema de justicia juvenil, bien de menores de 14 años directamente inimputables.

Como se señalaba al inicio de este epígrafe, las responsabilidades penales deben dirimirse a través de los diversos delitos tipificados en el código penal de manera general. Es habitual que en las conductas de acoso se acuda al delito contra la integridad moral recogido en el art.173.1 del Código Penal: infligir un trato degradante que menoscabe gravemente la integridad moral ${ }^{15}$. A través de este delito se castigarían todos aquellos comportamientos que vulneren la integridad moral protegida por el art.15 de la Constitución, que

13. Estas reflexiones se recogen, por ejemplo, en la Sentencia del Juzgado de Menores núm.1 de Palencia, núm. 61/13, cuando la Magistrada señala que nuestro ordenamiento carece de un derecho penal especial del adolescente, qué sí existe en países de nuestro entorno, que adapte los delitos que pueden cometer, como tampoco existe una doctrina penal específica sobre la autoría que comprenda la «actuación eminentemente grupal del adolescente» (FJ 3. ${ }^{\circ}$.

14. Respecto de la necesidad de una respuesta integral legal, véase LOSADA, Nazario; LOSADA, Ramiro; AlCÁZAR, Miguel Ángel; Bouso, José Carlos; GÓMEZ-Jarabo, Gregorio. «Acoso escolar: desde la sensibilización social a una propuesta de intervención. Reflexiones desde la legislación española», en Letras jurídicas, 4 (2007), pp. 11-15. Disponible en <http://letrasjuridicas.cuci.udg.mx/index.php/revista-numero-04-primavera-marzo-septiembre-de-2007>, consultado el 22-04-2015.

15. En esta exigencia de menoscabo grave es donde cobra especial relevancia uno de los elementos que suele usarse para caracterizar el acoso escolar, como es la reiteración de conductas. Desde el punto de vista penal, es precisamente esta reiteración la que, en muchos casos, permitirá que conductas que, si se analizaran de manera aislada o puntual no tendrían entidad suficiente como para realizar el tipo penal, puedan constituir base suficiente para afirmar su gravedad. 
proscribe, junto a la tortura, los tratos inhumanos y degradantes. El acoso escolar vulneraría -véase la jurisprudencia estudiada en la Instrucción-, esta última prohibición, a través de los actos susceptibles de causar angustia, terror y sentimientos de inferioridad que puedan suponer una humillación, envilecimientos y quebrar la resistencia física o moral. Asimismo, el art. 177 del Código Penal prevé la posibilidad del concurso de delitos si es que ha habido actos de lesiones físicas, de amenazas o coacciones, contrarios a la libertad sexual o a la propiedad privada, supuestos todos ellos habituales en el acoso escolar. Señala la Fiscalía en la citada Instrucción que, al estar guiada la sanción por los principios de tipicidad y ley cierta, pueden darse conductas típicas del acoso escolar que no encajen en ningún tipo penal y no puedan, por tanto, ser susceptibles de reproche en este sentido. Las conductas de aislamiento social, habituales en los casos de acoso escolar, quedarían fuera de la aplicación de la respuesta penal ${ }^{16}$. Esta última consideración nos acerca al objeto último de estas reflexiones: la respuesta penal no debe ser ni la única ni la primera respuesta. Existe consenso en la literatura respecto tanto de la necesidad de prevención como del papel primordial que el centro educativo, la comunidad escolar y la familia deben cumplir una vez identificado un supuesto de acoso escolar ${ }^{17}$. La variedad y peculiaridad de las conductas que pueden conformar una situación de este tipo, aspecto en el que ya se ha insistido, confirman estas reflexiones desde el punto de vista jurídico.

Debe tenerse en cuenta, por lo demás, que resulta especialmente difícil obtener en estos casos una sentencia que obligue a indemnizar si no ha habido previamente una condena penal, lo que puede condicionar el recurso a

16. Debe señalarse, para despejar errores, que en los casos más graves en los que, como sucedió con Carla, la víctima del acoso se suicida, eso no significa que quienes realizaron los actos de acoso escolar sean responsables de un delito de inducción al suicidio. Este tipo penal no reposa en el resultado, sino que está pensado para castigar aquellas conductas que tienen, como intención directa, incitar a la persona a suicidarse. Cuando no puede demostrarse que la intención -en este caso, de quien realiza acoso escolar- era la de influir en el ánimo de la persona para que se quitase la vida- la conducta no es constitutiva de dicho delito. Véase al respecto la primera de las sentencias del «caso Jokin», del Juzgado de Menores de San Sebastián (12-05-2005), Fundamento de Derecho 3. ${ }^{\circ}$

17. Por lo demás, quienes se han visto implicados en casos de acoso escolar, consideran que las medidas educativas impuestas son afrontadas por los menores como una verdadera sanción que les hace enfrentarse a sus responsabilidades. Si se repara en las medidas socio-educativas que se imponen en muchos casos, se verá que sus finalidades no son diferentes de las que propugnan quienes han investigado el tema del acoso escolar, al objeto de prevenir estos comportamientos: fomentar el respeto y la empatía por los demás, así como el aprendizaje de instrumentos de resolución de conflictos alternativos a la violencia. ¿Por qué no terminan de incorporarse al currículo educativo?

Feminismo/s 25, junio 2015, pp. 13-34 
dicha jurisdicción al objeto de garantizar una justa y necesaria indemnización por los daños sufridos, incluso en los casos menos graves en los que reaccionar frente a comportamientos violentos no pasaría necesariamente por la vía penal. Asimismo, resulta igualmente problemática cuando los responsables son menores de 14 años y, por tanto, inimputables ${ }^{18}$. Todo ello debería ser tenido en cuenta a la hora de abordar la regulación del acoso escolar.

El gran problema que subsiste, por último, es el de la eventual responsabilidad civil que pueda derivar de un caso de acoso escolar, ya sea por parte de los progenitores o tutores del menor que los ha cometido, ya sea por parte del centro educativo y, en último término, de la propia Administración pública ${ }^{19}$. En este sentido, y puesto que se pondrá el acento en el trascendental papel que debe jugar el centro escolar, parece necesario reivindicar una mayor seguridad jurídica para el personal del centro respecto de sus obligaciones ante los indicios de acoso escolar, pues sigue faltando un protocolo común que establezca, al menos, unas pautas mínimas, y que ofrezca certezas a los centros respecto de lo que se les puede exigir desde el punto de vista de la diligencia debida $^{20}$. Como señala la Fiscalía General del Estado, el hecho de que se inicie un expediente por la vía de la justicia juvenil no significa que el centro se inhiba y decline su responsabilidad.

18. La distinta respuesta jurídico-penal a los actos de acoso en función de la edad del menor, se desarrolla en FANJUL, José Manuel. «Visión jurídica del acoso escolar (bullying)», en la revista de la asociación de inspectores de Educación de España (ADIDE): Avances en Supervisión Educativa 17 (2012), disponible en:

$<$ http://www.adide.org/revista/images/stories/revista17/ase17_art03.pdf> consultado el 03-04-2015.

19. Véase al respecto FAnjul, José Manuel. «La responsabilidad civil del profesorado no universitario», en la Revista de la asociación de inspectores de Educación de España (ADIDE): Avances en Supervisión Educativa 14 (2011), disponible en:

<http://www.adide.org/revista/index.php?option=com_content\&task=view\&id=289\& Itemid $=70>$ consultado el 03-04-2015. En este trabajo se explica la vía de la responsabilidad civil extracontractual utilizada por los tribunales para exigir indemnizaciones en aquellos casos de acoso escolar en los que se haya considerado probada la actuación negligente por parte del centro.

20. Reflejo legal de las obligaciones de custodia es el Real Decreto 732/1995, de 5 de mayo, por el que se establecen los derechos y deberes de los alumnos y las normas de convivencia en los centros. En el Principado de Asturias, relevante desde el punto de vista del caso de Carla, debe tenerse igualmente en cuenta el Decreto 249/2007, por el que se regulan los derechos y deberes del alumnado y normas de convivencia en los centros docentes no universitarios sostenidos con fondos públicos del Principado. El propio título de la normativa nos pone sobre la pista del «enfoque de derechos» que se desarrollará en el epígrafe 4 de este trabajo, como principio orientador de la intervención ante el acoso escolar. 


\section{El menor como sujeto vulnerable}

\subsection{El menor como titular de derechos fundamentales}

La reivindicación de los menores de edad como sujetos titulares de derechos fundamentales ha ido cobrando intensidad en los últimos años, frente a las concepciones en las que la minoría de edad se equiparaba a una situación de incapacidad total para tomar decisiones sobre su propia vida e intereses, y la patria potestad se entendía como una situación de dominio sobre los hijos. Los tribunales han ido afirmando que el menor es titular de derechos, al margen de que la capacidad para ejercerlos de manera autónoma sea diferente y graduable en función tanto del derecho que esté en juego como de la edad y madurez de la persona. De esta manera, el principio del interés del menor que debe regir las decisiones de los progenitores o tutores del menor deja de ser un principio paternalista para entenderse como un principio orientado a facilitar el ejercicio de sus derechos por parte de los menores ${ }^{21}$. Desde este punto de vista se puede juzgar si las decisiones que los tutores tomen para complementar la falta de capacidad del menor, permitiendo un ejercicio heterónomo de sus derechos, respetan o no su propio interés. No debe ser casualidad, pues, que un objetivo fundamental de la educación sea transitar desde la heteronomía a la autonomía moral, tránsito íntimamente relacionado con la concepción de los menores como titulares de derechos fundamentales.

Este debe ser el criterio que presida las actuaciones de los sujetos implicados en los casos de acoso escolar ${ }^{22}$. Analizar las situaciones en las que se ven envueltos los menores desde un enfoque de derechos permite orientar las necesidades y las reacciones ante tales sucesos ${ }^{23}$. En primer lugar, deben tenerse en cuenta los derechos del menor que sufre el acoso, como son el

21. Es fundamental en este campo la aportación de ALÁEZ, Benito. Minoría de edad y derechos fundamentales, Madrid, Tecnos, 2003.

22. Por fortuna, este enfoque de derechos es el predominante en los últimos años en la actuación de numerosas instituciones públicas. Lo expresa con claridad un informe del Ararteko cuando afirma que «no solo es una cuestión de buena o mala educación. Es una cuestión de derechos, de derechos esenciales, puesto que afectan a la dignidad básica de las personas». Véase el Informe extraordinario del Ararteko sobre la situación en los centros de Educación Secundaria de la CAPV «Convivencia y conflictos en los centros educativos», 2006, pp. 21 y 27 y ss. Que las instituciones encargadas de la defensa de los derechos de la ciudadanía -el Defensor del Pueblo también se ha ocupado de este fenómeno- haya prestado tan gran atención a este asunto constituye, per se, una evidencia de la trascendencia de los derechos en juego.

23. Así por ejemplo, una de las grandes investigadoras de este problema señala que entre las estrategias de prevención de la agresión se encuentra la de «favorecer la identificación con el respeto a los derechos humanos». Véase DíAZ-AGUAdo, María José. «La violencia entre iguales en la adolescencia...». Op. cit., pp. 550-551.

Feminismo/s 25, junio 2015, pp. 13-34 
derecho a la vida, a la integridad física y moral, al honor, el derecho a la educación, y el derecho a la igualdad y no discriminación, del que se hablará con más detalle en el apartado siguiente. Todos estos derechos pueden verse afectados, y esta posibilidad es suficiente como para echar por tierra cualquier atisbo de tratar la situación de acoso como una situación «normal» entre adolescentes que debe servirles para «crecer» 0 «hacerse fuertes», así como para desterrar la idea de que lo mejor que puede hacerse es no intervenir al tratarse de un conflicto entre iguales ${ }^{24}$. Así lo han puesto de relieve los tribunales, al señalar que

El hecho de que este tipo de comportamientos hayan sido tradicionalmente tolerados, como incluidos en el acervo propio de la experiencia escolar, no les convierte en lícitos (...) Lo que ocurre es que en este ámbito no se lleva tanto camino recorrido como en otros casos de manifestación de la violencia... como puede ser la violencia intrafamiliar o incluso la violencia de género. Con este tipo de violencia tiene que ocurrir lo mismo que ha pasado con la violencia doméstica... dejar de considerarlo como algo inevitable y en cierta manera ajeno a las posibilidades de intervención del sistema penal, como problema de carácter estrictamente privado que debía ser solventado en el seno de las relaciones entre iguales, o cuando más en el ámbito de la disciplina escolar (...) Falta por conseguir en este ámbito, el convencimiento por parte de la sociedad de que este problema debe ser afrontado con un grado de tolerancia cero, de forma que ninguna clase de maltrato entre iguales debe ser admitido ni tolerado ${ }^{25}$.

Respecto de estas palabras, hay que señalar que cuando una de las partes se encuentra en situación de vulnerabilidad, no parece preciso hablar de un enfrentamiento entre iguales. De manera sintética, podría decirse que bien por razones cuantitativas - un alumno que se ve enfrentado a varios compañeros- bien por razones cualitativas -compartir algún rasgo identitario de algún colectivo minoritario o excluido, por ejemplo- una persona puede resultar vulnerable en

24. No se afirma que esta reacción sea generalizada y, en todo caso, ni mucho menos que sea exclusiva del centro escolar, pues como se refleja en el Informe de 2006 del Ararteko previamente citado, buena parte del alumnado considera habitual y justificado tanto las agresiones hacia sus compañeros/as, como el papel de testigo impasible que ni ayuda a quienes sufren los actos de acoso ni colabora con el profesorado. Op. cit., p. 33. Ahora bien, la reacción del centro es, lógicamente, fundamental, al ser sus miembros -y particularmente los docentes- quienes deben, en un primer momento, hacer frente a la situación y tomar conocimiento de lo que está sucediendo para poder valorar su gravedad y planificar la intervención.

25. Estas son las reflexiones de la magistrada en la Sentencia 61/13 del Juzgado de Menores de Palencia ya citada, frente a las opiniones de una parte del profesorado del centro escolar donde se había producido el caso de acoso escolar objeto de enjuiciamiento, y que aseveraban que lo ocurrido eran incidentes aislados «a los que no hay que dar mayor importancia», o bien "problemas de convivencia habituales», o ya «piques habituales entre niños».

Feminismo/s 25, junio 2015, pp. 13-34 
las relaciones entre quienes parecen ser sus «iguales» ${ }^{26}$. Esta reflexión encaja en las definiciones de acoso escolar que presentan como uno de los rasgos constitutivos la existencia de una situación de abuso de poder de la que la víctima no puede salir por sí sola. En todo caso, al menos un derecho de los alumnos implicados seguiría afectado: el derecho a la educación. En primer lugar, el derecho de la víctima, pues existe acuerdo entre los investigadores en señalar que es habitual que se vea afectado su rendimiento académico, pudiendo llegar incluso a abandonar los estudios para poder evitar, con ello, a quienes le agreden.

Pero la afectación del derecho a la educación desborda la perspectiva exclusivamente individual de la víctima. Dice el art.27 de la Constitución que el derecho a la educación «tendrá por objeto el pleno desarrollo de la personalidad humana en el respeto a los principios democráticos de convivencia y a los derechos y libertades fundamentales $»^{27}$. Parece evidente que el centro escolar es el primer sujeto obligado a realizar el derecho a la educación consagrado constitucionalmente. Difícilmente puede alegarse que se respeta este derecho si se permite que el alumnado se insulte, se amenace o recurra a la agresión en sus relaciones interpersonales. Esta finalidad del derecho a la educación es la piedra angular de este trabajo, y conduce a reflexionar sobre uno de los problemas que sistemáticamente se observan en los casos de acoso escolar: la presencia de patrones de género y la incapacidad para aceptar y respetar la diferencia como «motivos» de los actos de violencia. A la hora de analizar la responsabilidad del centro escolar frente al acoso se retornará sobre la centralidad del derecho a la educación.

\subsection{La violencia escolar en los menores, con especial atención a la violencia por} razón de género.

\subsubsection{Los patrones de género en el acoso}

El bullying esconde los aspectos de género de la violencia bajo una apariencia de neutralidad, actuando «como un eufemismo para comportamientos más

26. Véase en este sentido -el menor «diferente» como sujeto vulnerable- en las reflexiones de Blaya, Catherine; Debarbieux, Eric y LuCAS, Beatriz. «La violencia hacia las mujeres...». Op. cit., pp. 61-81. También en la citada Instrucción 10/2005 de la Fiscalía se recoge este concepto de alumno especialmente vulnerable (apartado 7.2).

27. Véanse los trabajos de Álvarez ÁlvareZ, Leonardo. «Education and pluralism. Towards a democratic theory of education in Europa», Intercultural Human Rights Law Review 6 (2011), pp. 349-378; y «Fines educativos y sociedades paralelas. Estructura y naturaleza normativa de los fines educativos en un sistema democrático», en PRESNO LINERA, Miguel Ángel; GuTIÉRREZ, Ignacio. (coords.). La inclusión de los otros: símbolos y espacios de la multiculturalidad. Granada, Comares, 2012, pp. 219-248. 
problemáticos como el racismo, la homofobia y el sexismo (...) desviando la responsabilidad legal que tiene la escuela de proporcionar un ambiente de aprendizaje seguro y equitativo $»^{28}$.

Este trabajo comparte la creencia de que el examen de la violencia relacionada con el género debe entenderse no solo como violencia dirigida contra las mujeres por razón de sexo y género, sino también la ejercida contra los hombres por no «cumplir» con el estereotipo de masculinidad propio de nuestras sociedades, y que es el complemento indispensable de la construcción de los roles o identidades de género femenino ${ }^{29}$. Al hacerlo así es posible obtener una mayor comprensión del fenómeno de la violencia escolar, en particular desde la perspectiva de género, incorporando la reflexión sobre las dificultades del alumnado para aceptar identidades sexuales que desafían la normatividad social hegemónica de su entorno, y que se reflejan en el uso peyorativo de tales identidades. En el caso de estudio, a Carla -ante una posible relación afectiva con otra chica- la insultaban utilizando el término «lesbiana» ${ }^{30}$. El

28. LEACH, Fiona; DunNE, Máiréad; SALVI, Francesca. «School-related Gender-based violence. A global review of current issues and approaches in policy, programming and implementation responses to School-related Gender-based violence (SRCGV) for the Education Sector», Background research paper prepared for UNESCO, 21 de enero de 2014, disponible en:

http://www.unesco.org/new/fileadmin/MULTIMEDIA/HQ/HIV-AIDS/pdf/SRGBV_ UNESCO_Global_ReviewJan2014.pdf > consultado el 26-03-2015.

29. Una vez más la referencia es al trabajo de DíAZ-AGUADO, María José, «La violencia entre iguales en la adolescencia... ». Op. cit., pp. 552 y ss., en el que recoge los resultados de las investigaciones sobre acoso escolar relativos a la relación entre violencia, género y sexismo, poniendo de relieve cómo la principal condición de riesgo no parece ser tanto el sexo como condición biológica sino «la identificación con el dominio de los demás, asociado al estereotipo masculino tradicional». Véanse también los trabajos de: LOMAS, Carlos. "¿La escuela es un infierno? Violencia escolar y construcción cultural de la masculinidad», Revista de Educación 342 (2007), pp. 83-101, destacando el papel de la educación para afrontar el vínculo entre masculinidad hegemónica y violencia escolar y social, lo que «obliga a trabajar... también con los agresores» (p.98); HERNÁNDEZ, Fernando; VIDIELla, Judit; HerRaIZ, Fernando; SANCHO, Juana María. «El papel de la violencia en el aprendizaje de las masculinidades», Revista de Educación 342 (2007), pp. 103-125; PeÑA, José Vicente; RodríGueZ, María del Carmen. «Identidades esquemáticas de género en la escuela: a propósito del primer aniversario de la muerte de Pierre Bordieu». Teoría de la Educación. Revista interuniversitaria 14 (2002), pp. 235263, en particular las pp. 246-256; Rodríguez, María del Carmen; PeÑA, José Vicente. «La investigación sobre el género en la escuela: nuevas perspectivas teóricas». Teoría de la Educación. Revista interuniversitaria 17 (2005), pp. 25-48.

30. Esto concuerda con los estudios que señalan que hasta un $50 \%$ de jóvenes lesbianas, gays o bisexuales declaran haber sido insultados en la escuela secundaria, como los de D'Augelli o Rivers, citados por BlaYA, Catherine; DeBARBIEUX, Eric y LuCAS, Beatriz. «La violencia hacia las mujeres...». Op. cit., p. 72.

Feminismo/s 25, junio 2015, pp. 13-34 
desafío de los «ideales hegemónicos» por parte de «otras feminidades» (e igualmente sucedería en el caso de «otras masculinidades») puede incurrir «en costes emocionales y sociales, siendo calificados de marginados o subordinados ${ }^{31} \gg$. Así, los estudios más recientes sobre violencia escolar relacionada con el género, una vez superada la fase inicial centrada exclusivamente en la violencia heterosexual, han pasado a incluir la

violencia homofóbica contra el alumnado (y profesorado) lésbico, gay, bisexual o trans-género, basada en su orientación sexual (...). La violencia homofóbica... se compone de formas de violencia simbólica que también se producen por la exclusión, dentro del marco del discurso y el currículo educativo, de cualquier versión del género, la identidad sexual y las relaciones sexuales que no sean las versiones hetero-normativas ${ }^{32}$.

Estas expresiones de la violencia escolar constituyen una manifestación de la incapacidad por parte de quienes la ejercen de asumir -y en este sentido, respetar- las diferencias, la diversidad de identidades (culturales, nacionales, étnicas, sexuales, etc.), características de cualquier sociedad pero que se hacen más visibles con las experiencias de la globalización y la ruptura -gracias al principio democrático- de la falacia de la homogeneidad social. No en vano se ha afirmado que uno de los factores de riesgo relacionado con el acoso escolar es el mero hecho de ser diferente ${ }^{33}$. Frente a estas conductas de violencia, la respuesta del centro educativo es fundamental, debiendo abordar el conflicto desde el punto de vista de los derechos de los menores víctimas del acoso, y de los valores democráticos de respeto, tolerancia y pluralismo. Lo contrario sería tanto como sumar, a las conductas de acoso del alumnado, una actitud de homofobia institucional, al permitir la violación de los derechos de estas personas ${ }^{34}$.

31. Véase al respecto Rodríguez, M. ${ }^{a}$ del Carmen; PeÑA, José Vicente. «Identidad de género y contexto escolar: una revisión de modelos», Reis (Revista Española de Investigaciones Sociológicas), 112/05, p. 179.

32. Ibídem, p. 14. Se ve reafirmado, por ejemplo, en el Informe Ejecutivo sobre el Maltrato entre iguales en Euskadi previamente citado.

33. Dice el Ararteko en el citado Informe de 2006 que lo que claramente parece un factor de riesgo es el hecho de ser diferente en cualquier sentido, ver p. 43 y la bibliografía que cita. En este sentido, y centrado en las diferencias por razón de orientación sexual, véase el trabajo de BlaYA, Catherine; Debarbieux, Eric y LuCAS, Beatriz. «La violencia hacia las mujeres y hacia otras personas percibidas como distintas a la norma dominante: el caso de los centros educativos». Op. cit., en particular pp. 70 y ss. Respecto de los chicos, DíAz-AGUADO, María José, «La violencia entre iguales en la adolescencia...». Op. cit., p. 551, y la bibliografía que cita.

34. La Sentencia de la Audiencia Provincial de Guipúzcoa, de 15 de julio de 2005, que conoció el recurso de apelación en el «caso Jokin», habla de la necesidad de un marco de «contención de los menores», que en primer término debe reposar en la familia.

Feminismo/s 25, junio 2015, pp. 13-34 


\subsubsection{Prevención de la violencia}

Si en los fenómenos de violencia escolar están presentes, entre otros, patrones de género, y si además se ven afectados derechos fundamentales de (todos) los menores implicados, es evidente que el centro escolar debe ser la herramienta principal al abordar estas situaciones de conflicto, usando como instrumento precisamente lo que constituye su razón de ser: la educación. Asumiendo el carácter socialmente construido de las identidades de género, la escuela (y el instituto) es uno de los principales centros de socialización y, por lo tanto, de construcción de identidades, sobre todo grupales ${ }^{35}$. Su objetivo ha de ser una educación crítica que permita revisar tanto «los elementos sociales que sustentan el imaginario colectivo, como los valores individuales que configuran la personalidad de los futuros hombres y mujeres, en los que ya no podrá estar presente la desigualdad ${ }^{36}$.

Los supuestos de violencia escolar no son casos de violencia de género en el sentido previsto por el ordenamiento jurídico español, que afronta el problema de la violencia en el marco de las relaciones afectivas de pareja. Pero sí que está presente el género en el sentido explicado al abordar la presencia de patrones de género en la violencia escolar ${ }^{37}$. Y desde ese punto de vista, es fundamental hacer referencia al Capítulo I de la LO 1/2004, de medidas de protección integral contra la violencia de género, que recoge entre sus fines el respeto de los derechos, la igualdad de hombres y mujeres y la resolución pacífica de los conflictos. Es igualmente relevante la previsión de la LO 3/2007, para la igualdad efectiva de mujeres y hombres, cuyos arts. 23 y 24 señalan la obligación de que la educación incorpore entre sus fines

Resulta muy edificante el análisis que realiza de la reacción de las familias de los menores procesados, que no solo re-victimiza a Jokin, sino que «desresponsabiliza a los menores..., vaciando de contenido educativo a una respuesta que necesariamente debe vertebrarse en torno a la visibilidad del sufrimiento causado..., y la responsabilización por la humillación y el daño psíquico... para protagonizar una vida adulta guiada...por los principios de libertad, dignidad y responsabilidad» (FJ $5 .^{\circ}$ ).

35. El debate sobre el proceso de construcción de la identidad de género está lejos de estar cerrado, sin que ello signifique negar la importancia de la dimensión social / colectiva (como es la escuela) en dicha identidad. Véase al respecto, a modo de ejemplo, una revisión esquemática de las teorías de la socialización de roles de género frente a las teorías post-estructuralistas feministas en: ROdRíGUEZ, M. ${ }^{a}$ del Carmen; PeÑA, José Vicente. «Identidad de género...». Op. cit., pp. 165-194.

36. LORENTE, Miguel. «Violencia de género, educación y socialización: acciones y reacciones», Revista de Educación 342 (2007), p. 29.

37. La relación entre la violencia contra las mujeres y el bullying se estudia también en ZEMBYLAS, Michalinos; KALOYIROU, Christalla. «Emociones y violencia contra las mujeres y otros grupos. Implicaciones pedagógicas», Revista de educación 342 (2007), pp. $37-59$.

Feminismo/s 25, junio 2015, pp. 13-34 
«la educación en el respeto de los derechos fundamentales» y la educación «en la igualdad de derechos y oportunidades entre mujeres y hombres», así como la obligación de «eliminar y rechazar los contenidos y comportamientos sexistas». Afrontar los casos de violencia escolar en los que estén presentes patrones de género, como era el caso de Carla, permitirá resolver no solo la situación de acoso, sino que avanza igualmente en la finalidad prevista por ambas leyes. No se pueden desconectar las conductas de acoso escolar que evidencian una incapacidad para respetar las identidades no normativizadas, de la existencia de violencia de género en el marco de las relaciones de pareja. No se quiere decir, obviamente, que quien de adolescente realiza conductas de acoso escolar acabe realizando conductas de violencia de género en sus relaciones afectivas. Simplemente se trata de poner de relieve la trascendencia de los patrones de género en muchos casos de acoso escolar, y el hecho de que abordar estas situaciones desde la comunidad educativa sin duda puede aportar herramientas a la hora de aceptar la diversidad, que también ayudará a establecer relaciones sentimentales respetuosas de la individualidad de cada miembro de la pareja y a no recurrir, en caso de conflicto, a la violencia.

No son pocos, en definitiva, los rasgos que la violencia de género en sentido estricto comparte con la violencia escolar: la citada «conspiración del silencio»; el haberlos considerado tradicionalmente como asuntos que pertenecía a la esfera privada de las personas; el haber entendido que -mientras no excediera límites demasiado graves- formaba parte de la «normalidad»; así como la existencia de una doble victimización, al focalizar la atención sobre la supuesta falta de reacción de las víctimas. Por suerte, estamos llegando a un tiempo nuevo en la conciencia social, en la que cada vez más ambos asuntos se entienden no como fenómenos aislados o puntuales, sino como situaciones estructurales que hunden sus raíces en la desigualdad. Afrontar las situaciones de violencia desde el ámbito escolar es parte de esta estrategia global ${ }^{38}$. Como señala el Ararteko en el citado informe de 2006, aunque «la escuela, por sí sola, no puede desterrar la violencia de la sociedad (...) sí puede prevenirla, reducir su presencia o sus consecuencias, y ofrecer modelos alternativos de relación».

38. PANCHÓN E IGLESIAS, Carme. «Modelos educativos alternativos que conllevan el reconocimiento y el respeto por el otro». Revista de Educación 342 (2007), pp. 147-166. 


\section{La responsabilidad del centro escolar. El «acoso 2.0»}

\subsection{La convivencia democrática como objeto del derecho a la educación}

Como ya se ha puesto de relieve, la obligación de que el centro escolar no se inhiba ante estas situaciones de bullying deriva en un primer momento de los derechos de los menores implicados.

En este sentido, se indicaba en páginas anteriores que uno de los derechos en presencia es el propio derecho a la educación, que tiene por objeto constitucional no solo el aprendizaje de unas materias sino también la formación de ciudadanos respetuosos con los derechos y libertades fundamentales de los demás, esto es, el derecho a ser educado en los valores democráticos de convivencia. La escuela debe ser, ante todo, una escuela de ciudadanos libres y democráticos que respeten el pluralismo que, según el preámbulo de nuestra Constitución, es uno de los valores superiores del ordenamiento, inherente al propio concepto de democracia, y fundamento por tanto de nuestro derecho a ser diferentes sin sufrir menoscabos o perjuicios por ello ${ }^{39}$. Esta afirmación encuentra respaldo en textos de organismos internacionales fundamentales en el ámbito educativo, como puede ser el conocido como Informe Delors de la UNESCO, que señala que también es tarea del sistema educativo garantizar nuevas «alfabetizaciones» relacionadas con el desarrollo moral, emocional y social.

La prevención del acoso escolar debe ser considerado un marco óptimo para hacer efectiva una educación en valores desde la perspectiva de los derechos constitucionales y la convivencia democrática, favoreciendo la cohesión e integración de todo el alumnado, con especial atención a quienes presenten factores de vulnerabilidad, tal y como ya se ha señalado; generando una

39. Esta afirmación encuentra respaldo en la ya citada Sentencia del Juzgado de Menores de Palencia, cuando al establecer la responsabilidad solidaria de los padres, tutores y demás guardadores prevista por la LO 5/2000, reguladora de la Responsabilidad Penal del Menor, la magistrada señala (FJ $7 .^{\circ}$ ) que es una consecuencia derivada de «la falta de diligencia en la vigilancia, custodia y educación de los menores (...). Esta responsabilidad nacería de su propia conducta, distinta e independiente de la del menor, consistente en su omisión de su deber de educar al citado menor...» (la cursiva es mía). La obligación suele extenderse a los centros educativos por la vía de la responsabilidad extra-contractual derivada del art.1903 del Código Civil, por vulnerar el citado RD 732/1995, cuando no se haya garantizado suficientemente los derechos de los alumnos y no se haya impedido la comisión de hechos contrarios a las normas de convivencia de los centros. Frente a esta práctica jurídica, se comparten las opiniones de la Fiscalía en su Instrucción 10/2005 cuando señala que, para facilitar el proceso y la exigencia de responsabilidades, en la figura del guardador (de hecho) previsto por la LORPM citada deberían entenderse incluidos los centros docentes. 
reflexión crítica sobre los prejuicios que existen en la sociedad respecto de los colectivos más vulnerables y en muchas ocasiones, socialmente excluidos ${ }^{40} \mathrm{y}$, por último, afirmando una cultura de la no-violencia que enseñe al alumnado métodos de resolución de conflictos alternativos a la violencia ${ }^{41}$.

\subsection{El problema del ciber-acoso y el nexo escolar}

En último lugar, debe tenerse presente que el centro escolar es el nexo que une a las personas implicadas en la situación de bullying, y este nexo ha de ser considerado el de mayor relevancia a la hora de entender la obligación de actuación del centro. Que las conductas de acoso se realicen no sólo en el espacio físico del centro, sino en el espacio virtual de las redes sociales dificulta que se conozca con detalle los hechos, o en todo caso, puede exigir la intervención de otros sujetos así como una investigación que vaya más allá de la observación dentro del colegio. Pero estas dificultades deben llevarnos a analizar las herramientas que pueden necesitar los centros educativos para afrontar estas situaciones, sin que en ningún caso deban ser usadas para negar la responsabilidad del centro en los casos de violencia escolar.

El uso de redes sociales no hace que el conflicto sea externo al centro, pues la relación de compañeros de colegio / instituto es primordial, toda vez que explica la necesaria interdependencia entre víctima y agresores, algo que no se daría si no existiera dicha relación ${ }^{42}$. La relevancia del nexo escolar $-\mathrm{y}$, por tanto, el papel fundamental que el centro debe cumplir en esta situación-se ve reforzada si se tiene en cuenta que las conductas de maltrato entre iguales son fenómenos de grupo, vinculadas a las propias dinámicas de los colectivos dentro de la clase o del contexto más amplio del centro escolar ${ }^{43}$. La relación escolar es el contexto para las situaciones de acoso, la que posibilita

40. En esta vía insisten AlonQueo, Paloma; BARRIo, Cristina del. «The incidence and meaning of peer bullying in a multi-ethnic school». En Ross, A. (ed.), A Europe of Many Cultures. Londres, 2003, pp. 113-121.

41. De nuevo Díaz-AguAdo, María José. «La violencia entre iguales en la adolescencia...». Op. cit., pp. 554 y ss.

42. Respecto de la relevancia del vínculo escolar, véase ANDRÉS GÓMEZ, Soledad; BARRIOS, Ángela. "De la violencia a la convivencia en la escuela: el camino que muestran los estudios más recientes». Revista Complutense de Educación. 20, 1 (2009), p. 207.

43. Este aspecto se pone de relieve en gran parte de los estudios citados. Como aspecto central de la investigación, véase el trabajo de LuCAS, Beatriz; PULIDO VALERO, Rosa; SOLBES, Irene. «Violencia entre iguales en Educación Primaria: el papel de los compañeros y su relación con el estatus sociométrico». Psicothema 23, 2 (2011), pp. 245-251. La dimensión grupal cobra gran relieve también en la jurisprudencia. La sentencia citada del Juzgado de Menores de San Sebastián (caso Jokin) señala como «la importancia del grupo es fundamental (...) nunca actuaban individualmente» (FJ 9. ${ }^{\circ}$ ) 
que se den y la que explica las graves consecuencias del mismo, toda vez que posiciona a la víctima dentro de la comunidad, bien excluyéndola, bien marcándola con determinados rasgos considerados negativos. Se establecen así relaciones de poder y dominación sobre la misma, inherentemente contrarias a la idea de dignidad. Los expertos ponen de relieve que los enfoques «clínicos», es decir, los que tratan exclusivamente el problema entre víctima y agresor, desconocen este perfil social fundamental en estos procesos; por eso la institución educativa debe ser clave en su resolución, canalizando la participación de toda la comunidad educativa implicada, y afrontándolo como un problema de dicha comunidad ${ }^{44}$.

\section{Conclusiones}

En este trabajo se ha intentado exponer algunas de las cuestiones jurídicas de mayor interés a la hora de abordar los problemas relacionados con la violencia escolar, a partir de reflexiones suscitadas a raíz del caso de Carla. La respuesta penal no debe ser ni la única ni la principal, pues no puede dar satisfacción a la complejidad de factores presente en los casos de acoso. Concentrar la atención en la vía penal ha descuidado el papel fundamental que el derecho constitucional a ser educados en el respeto y la tolerancia a los derechos y libertades de las demás personas debe jugar en el marco de los centros educativos. El alumnado debe aprender a respetar la diversidad -incluida la de sexo y género, al ser los factores vinculados con el género y con la orientación sexual habituales «motivos» de acoso escolar-, así como métodos de resolución de conflictos alternativos a la violencia. Ser plenamente conscientes de que hay derechos fundamentales de los menores implicados en los casos de violencia escolar debe permitir un mejor enfoque de la reacción ante dichos sucesos, por parte tanto del centro educativo, de la administración pública y de las familias y el alumnado. El centro educativo ha de ser el referente principal, dada su función primordial en el derecho a la educación, y la generalización del uso de nuevas tecnologías en los comportamientos de acoso no puede ser utilizado para desatender dicha responsabilidad, pues en ese caso se estaría desconociendo que un aspecto central en estos supuestos es, precisamente, el nexo escolar.

44. ORTEGA-RUIZ, Rosario; NúÑEZ, José Carlos. «Bullying and cyberbullying: Research and intervention at school and social contexts». Psicothema 24, 4 (2012), pp. 603-607.

Feminismo/s 25, junio 2015, pp. 13-34 


\section{Referencias bibliográficas}

AlÁEz, Benito. Minoría de edad y derechos fundamentales. Madrid, Tecnos, 2003.

AlONQUeO, Paloma; BARRIO, Cristina del. «The incidence and meaning of peer bullying in a multi-ethnic school». En Ross, A. (ed.), A Europe of Many Cultures. Londres, 2003, pp. 113-121.

Álvarez, Leonardo. «Education and pluralism. Towards a democratic theory of education in Europa», Intercultural Human Rights Law Review 6 (2011), pp. 349-378.

ÁLVAREZ, Leonardo. «Fines educativos y sociedades paralelas. Estructura y naturaleza normativa de los fines educativos en un sistema democrático». En Presno Linera, Miguel Ángel; Gutiérrez GutiÉRrez, Ignacio. (coords.), La inclusión de los otros: símbolos y espacios de la multiculturalidad. Granada, Comares, 2012, pp. 219-248.

Álvarez-García, David; NúÑez-PÉrez, José Carlos; Álvarez-PÉrez, Luis; DobARRO-GonZÁlez, Alejandra; RodríGueZ-PÉrEZ, Celestino; GonZÁlez-Castro, Paloma. «Violencia a través de las tecnologías de la información y la comunicación en estudiantes de secundaria». Anales de psicología 27, 1 (2011), pp. 221-231.

Álvarez-García, David; Dobarro, Alejandra; Álvarez, Luis; NúÑez, José C; Rodríguez, Celestino. «La violencia escolar en los centros de educación secundaria de Asturias desde la perspectiva del alumnado». Educación XXI 17.2 (2014), pp. 337-360.

ANDRÉS, Soledad; BARRIOS, Ángela. «De la violencia a la convivencia en la escuela: el camino que muestran los estudios más recientes». Revista Complutense de Educación 20, 1 (2009), pp. 205-227.

ARARTEKO, Informe extraordinario sobre la situación en los centros de Educación Secundaria de la CAPV "Convivencia y conflictos en los centros educativos", 2006.

Blaya, Catherine; Debarbieux, Eric y LuCAs Molina, Beatriz. «La violencia hacia las mujeres y hacia otras personas percibidas como distintas a la norma dominante: el caso de los centros educativos». Revista de Educación 342 (2007), pp. 61-81.

CÁCERES, María Pilar; AlOnso, Santiago; GARrote, Daniel. «Aportaciones para el estudio de la violencia escolar desde una perspectiva interdisciplinar desde el ámbito universitario, escolar, familiar y social», Ensayos 16 (2008), pp. 221-236.

CAlvete, Esther; ORUE, Izaskum; EstéVez, Ana; Villardón, Lourdes; PAdilla, Patricia. «Cyberbullying in adolescents: Modalities and aggressors' profile». Computers in Human Behaviour 26 (2010), pp. 1128-1135. 
DEFENSOR DEL PUEBLO. Informe: «Violencia escolar: el maltrato entre iguales en la Educación Secundaria Obligatoria 1999-2006», Madrid, 2007.

FANJUL, José Manuel. «La responsabilidad civil del profesorado no universitario», en la Revista de la asociación de inspectores de Educación de España (ADIDE): Avances en Supervisión Educativa 14, mayo 2011, disponible en $<$ http://www.adide.org/revista/index.php?option=com_content\&task=view\& $\mathrm{id}=289 \&$ Itemid $=70>$ consultado el 03-04-2015.

FANJUL, José Manuel. «Visión jurídica del acoso escolar (bullying)», en la Revista de la asociación de inspectores de Educación de España (ADIDE): Avances en Supervisión Educativa 17, noviembre 2012, disponible en: <http://www. adide.org/revista/images/stories/revista17/ase17_art03.pdf > consultado el 03-04-2015.

FÉliX-Mateo, Vicente; Soriano-Ferrer, Manuel; Godoy-Mesas, Carmen; SANCHO-VICENTE, Sonia. «El ciberacoso en la enseñanza obligatoria». Aula Abierta 38, 1 (2010), pp. 47-58.

GREENE, M.; Robles, O.; STOUT, K. y SuVILAAKSO, T. «A girls right to learn without fear: working to end gender-based violence at school». Woking: Plan international, 2013, disponible en:

<http://plan-international.org/about-plan/resources/publications/campaigns/ summary-a-girls-right-to-learn-without-fear/working-together-end-violence>, consultado el 11-03-2015.

HernándeZ, Fernando; Vidiella, Judit; Herraiz, Fernando; SAnCHO, Juana María. «El papel de la violencia en el aprendizaje de las masculinidades», Revista de educación 342 (2007), pp. 103-125.

InSTITUTO VASCO DE EVALUACIÓn E INVESTIGACIÓn EDUCATIVA. Informe ejecutivo: El maltrato entre iguales en Euskadi, 2012.

LEACH, Fiona; DunNE, Máiréad; SALVI, Francesca. «School-related Gender-based violence. A global review of current issues and approaches in policy, programming and implementation responses to School-related Gender-based violence (SRCGV) for the Education Sector», Background research paper prepared for UNESCO, 21 de enero de 2014, disponible en:

<http://www.unesco.org/new/fileadmin/MULTIMEDIA/HQ/HIV-AIDS/pdf/ SRGBV_UNESCO_Global_ReviewJan2014.pdf> consultado el 26-03-2015

LI, Qing. «New bottle but old wine: A research of cyberbullying in schools», Computers in Human Behaviour 23 (2007), pp. 1777-1791.

LosadA, Nazario; LosadA, Ramiro; AlCÁZAR, Miguel Ángel; Bouso, José Carlos; GÓMEZ-JARABO, Gregorio. «Acoso escolar: desde la sensibilización social a una propuesta de intervención. Reflexiones desde la legislación española». Letras jurídicas 4 (2007), pp. 1-16. Disponible en < http://letrasjuridicas. cuci.udg.mx/index.php/revista-numero-04-primavera-marzo-septiembrede-2007>, consultado el 22-04-2015. 
LOMAS, Carlos. «¿La escuela es un infierno? Violencia escolar y construcción cultural de la masculinidad». Revista de Educación 342 (2007), pp. 83-101.

LORENTE, Miguel. «Violencia de género, educación y socialización: acciones y reacciones». Revista de Educación 342 (2007), pp. 19-35.

Lucas, Beatriz; Pulido Valero, Rosa; Solbes Canales, Irene. «Violencia entre iguales en Educación Primaria: el papel de los compañeros y su relación con el estatus sociométrico», Psicothema 23, 2 (2011), pp. 245-251.

Ortega, Rosario; Calmaestra, Juan; Mora Merchán, Joaquin. «Cyberbullying». International Journal of Psychology and Psychological Therapy 8, 2 (2008), pp. 183-192.

ORTEGA-RUIZ, Rosario; NúÑEZ, José Carlos. «Bullying and cyberbullying: Research and intervention at school and social contexts». Psicothema 24, 4 (2012), pp. 603-607.

PANCHÓN e IGLESIAS, Carme. «Modelos educativos alternativos que conllevan el reconocimiento y el respeto por el otro». Revista de Educación 342 (2007), pp. 147-166.

Peña Calvo, José Vicente; Rodríguez, María del Carmen. «Identidades esquemáticas de género en la escuela: a propósito del primer aniversario de la muerte de Pierre Bourdieu». Teoría de la Educación. Revista interuniversitaria 14 (2002), pp. 235-263.

Rodríguez, M. ${ }^{a}$ del Carmen; PeÑA, José Vicente. «Identidad de género y contexto escolar: una revisión de modelos». Reis (Revista Española de Investigaciones Sociológicas) 112 (2005), pp. 166-194.

Rodríguez, M. ${ }^{a}$ del Carmen; PeÑA, José Vicente. «La investigación sobre el género en la escuela: nuevas perspectivas teóricas». Teoría de la Educación. Revista interuniversitaria 17 (2005), pp. 25-48.

TOKUNAGA, Robert S. «Following you home from school: A critical review and synthesis of research on cyberbullying victimization». Computers in Human Behaviour 26 (2010), pp. 277-287.

ZEMBYLAS, Michalinos; KAlOYIROU, Christalla. «Emociones y violencia contra las mujeres y otros grupos. Implicaciones pedagógicas». Revista de educación 342 (2007), pp. 37-59. 